\title{
Recent developments in quantitative SERS moving towards absolute quantification
}

Royston Goodacre ${ }^{1,}{ }^{*}$, Duncan Graham ${ }^{2}$ and Karen Faulds ${ }^{2}$

${ }^{1}$ The Manchester Institute of Biotechnology, School of Chemistry, University of Manchester, 131 Princess Street, Manchester, M1 7DN, U.K.

${ }^{2}$ Centre for Molecular Nanometrology, Department of Pure and Applied Chemistry, Technology and Innovation Centre, University of Strathclyde, 99 George Street, Glasgow, G1 1RD, U.K.

*Corresponding author: roy.goodacre@manchester.ac.uk

\section{SUMMARY}

Surface enhanced Raman scattering (SERS) generates molecularly specific fingerprints of analytes and when the experimental conditions are carefully controlled this is highly quantitative. This review critiques the development of quantitative SERS from simple univariate assessment of single vibrational modes to multivariate analysis of the whole spectrum for improved quantification. SERS has also been developed for direct multiplex detection and quantification of multiple analytes and this is also discussed, as is the need for LC-SERS for analyte separation should multivariate chemometric approaches fail to effect quantification. Finally, to effect absolute quantification with SERS, the concepts of isotopologues is introduced along with the standard addition method (SAM) and suitable examples that have been developed and exploited these techniques are presented. We believe that SERS will be routinely used for quantitative analysis and it is only a matter of time before this technique translates from the laboratory to the clinical environment. 


\section{THE NEED FOR QUANTIFICATION}

In all areas of science there is a need for absolute quantification of analytes of interest. This may be within the context of environmental science where the level of a toxicant polluting an ecological system must be below expected levels (usually set by a regulating authority in that country or region). Within food it is essential to know the levels of any pesticide or fungicide, as well as any chemical contaminant that may be present naturally or artificially by trying to make the food look more appealing [1]; for example, certain foods may contain the banned azo dye Sudan I which, along with Sudan III and IV, have been branded as category 3 carcinogens by the International Agency for Research on Cancer [2] and these dyes are often used to make chili or curry look brilliant red. Of course within medicine it is essential that key biomarkers of disease are assessed in a robust and reproducible manner - there can be no error in measurement here. By way of example, in clinical biochemistry when testing for Type II diabetes using the oral glucose tolerance test [3], the measurement of glucose in blood plasma must be reported in $\mathrm{mmol} / \mathrm{L}$ (or $\mathrm{mg} / \mathrm{dL}$ ) and the normal glucose concentration range for a fasted individual is expected to be 3.3 to $5.5 \mathrm{mmol} / \mathrm{L}$ (60 to $100 \mathrm{mg} / \mathrm{dL}$ ). In drugs testing accurately determining the level of a drug and its metabolites (so called xenometabolites) in blood or urine are needed either for illicit substance detection or for personalised medicine where it is important to know the pharmacodynamics of drug clearance.

Whatever analytical approach is used for measurement the output must be the same and must be reported in units that other scientists and non-cognoscenti understand. A cautionary reminder of what happens when this goes wrong is the Mars Climate Orbiter that on 23 September 1999 failed to insert into orbit around Mars, but rather crashed into the surface of this planet. This was because the design specification in the ground software was in US customary units (pound force-seconds) rather than SI units (newton-seconds); an expensive avoidable and somewhat embarrassing mistake [4].

Surface enhanced Raman spectroscopy (SERS) and the closely related Surface enhanced resonance Raman spectroscopy (SERRS) are analytical methods that are constantly being developed to measure a range of biologically-relevant molecules. These target molecules may be DNA, RNA or other nucleic acid sequences. While proteins and metabolites are also molecules that need accurate quantitative assessments and these too can be quantified using SERS-based analyses.

Whilst there are investigations that have reported SERS analysis directly on blood that could be used for stratification of people with disease from healthy individuals [5], as these are fingerprinting based and lack molecular resolution; that is to say, the SERS spectra from such a complex (bio)chemical mixture will be a combination of many molecules measured within these biofluids and so it is very difficult to assign bands to specific chemical entities without prior separation (e.g., by chromatography). We believe that the true power in SERS is for targeting molecules that are known to be useful for addressing a particular clinical acumen. Within this context these assays tend to be highly specific for 
the determinand of interest and are used when a known biomarker has clinical utility.

Figure 1 describes a typical biomarker pipeline from concept to clinical delivery [6]. After the clinical decision is made to look for new biomarkers for patient stratification a biomarker discovery phase is first undertaken. This is typically performed with small numbers of individuals and so needs to be repeated in a second cohort of different people to validate the initial study. At this stage the biomarker (nucleic acid, protein, metabolite) is unknown and so this is analytically intensive as mass spectrometry (MS) usually linked to prior chromatographic separation (viz. GC-MS and/or LC-MS) is the technology used in these analyses; these typically take 30 min per analysis type. MS has great appeal here as, along with NMR spectroscopy (on isolated molecules), allows structural elucidation and hence identification of targeted molecules [7]. At the end of this process a list of candidate molecules is generated which needs to be validate in larger cohorts before ready for use within the clinic. At this stage in addition to larger sample numbers there is no need to use expensive analytical techniques like GC-MS, LC-MS or NMR as these lack sample throughput. What is needed is a technique that is easy to use, potentially portable for at patient or bedside use and one that has chemical specificity for the target molecules of interest and is of course highly quantitative. Such portable point-and-shoot techniques [8] are ideal for personalized medicine where there is a need for at home testing [9], and as indicated in Figure 1 this is where SERS is best used as it addresses all the needs in terms of speed, high sample numbers, and when developed appropriately is highly specific and quantitative for target analytes.

\section{A BRIEF INTRODUCTION TO SERS AND QUANTITATIVE ANALYSIS}

Surface-enhanced Raman scattering (SERS) is a vibrational spectroscopic method that was discovered in 1974 which uses a metal surface to boost the Raman signal overcoming its rather weak output $[10,11]$; typically in Raman only 1 in $10^{6}-10^{8}$ photons undergo an inelastic light scattering event so collection times are long. SERS utilises roughened metal substrates, which may be roughed electrodes, thin films or nanoparticles (NPs). With respect to quantification NPs colloids are mainly used as these allow averaging of the signal during collection and hence improved quantification [12].

The use of NPs gives rise to large enhancements of the Raman signal and these are typically in the order of $10^{4}-10^{6}$, and when combined with an additional enhancement based on resonance between the analyte and excitation wavelength, even single molecule detection is possible [13]. There are also reports of single molecule detection in non-resonant molecules [14]. There is much debate on the underlying mechanisms that are responsible for SERS (see papers in Themed Collection: Surface enhanced Raman Scattering (2017) Faraday Discussions 205, 1-626). The dominant process is an electromagnetic (EM) effect, and this occurs when the electrons on a roughened metal surface (usually of $\mathrm{Ag}$ or $\mathrm{Au}$ for SERS, although others are possible) are excited by an incident laser light creating a localised surface plasmon (LSP) and any analyte 
that interacts with the LSP has an amplification of the EM field and hence enhancement of selective bands in SERS $[15,16]$. Another contribution to SERS, although at a lower level, is the chemical effect. This is based on a chargetransfer mechanism when the analyte forms a chemical bond with the metal surface, and there is a transfer of electrons from the metal to the surface and vice versa. This gives rise to increased molecular polarisability and thus also enhances the Raman signal [17].

Although the exact mechanisms are yet to be fully elucidated, therefore one cannot yet readily go from a computational modeling approach to generate a theoretical SERS spectrum that predicts laboratory-based investigations, this has not stopped SERS being used in a vast area of applications. For an excellent recent review on where SERS is used within biomedical applications the reader is directed here [18].

SERS is based on Raman spectroscopy and Raman (as the underlying technique) provides a signal that scales linearly with the number of molecules under investigation. Thus quantitative analyses can be conducted based on the band area for a specific molecular vibration, as well as band position and band shape [19]. SERS has also been used for quantitative analysis (vide infra) and as is the case in inherent/normal Raman, the band area(s) for specific molecules of interest also increase when more molecules are present within the interrogation voxel. However, it should be noted that as enhancement is greatest when molecules are adsorbed directly onto the metal surface or less than $1 \mathrm{~nm}$ away from the surface, quantitation is dependent on analyte concentration and surface coverage, as well as orientation of the molecule on the metal surface which may affect the SERS spectrum due to relative distance of different vibrational modes within a molecule from the LSP. The slope of the calibration graph is different in the regions above and below monolayer coverage of the metal surface and this should be taken into account when optimizing a detection approach [20].

Therefore, in order for an analytical technique to provide a quantitative output, that can be used to determine the level of an analyte, with a real unit (i.e. $\mathrm{mg} / \mathrm{mL}$ and not some arbitrary instrument response), it must first be calibrated. Calibration is commonplace for all detectors. Liquid chromatography-mass spectrometry (LC-MS), gas chromatography using a flame ionisation detector (GC-FID), or mass spectrometry (GC-MS), nuclear magnetic resonance (NMR) spectroscopy, electrochemical detection (ECD), etc. are not quantitative unless the detector response is related to the concentration of an analyte under investigation. And the same is true for SERS.

The most common method of calibration used in SERS is to take a well-defined SERS/Raman peak and first calculate the peak height or peak area; peak area tends to give more accurate results but this depends on the baseline and therefore sometimes peak heights are preferred, where it is usually easier to subtract the background contribution from the baseline. This is then plotted against the concentration of a target analyte and a calibration curve is constructed. The number of points within the linear range of this calibration plot should be at least 5 different concentration levels of the target analyte. If a full 
calibration is performed the calibration curve will be S-shaped: at low concentrations no analyte is detected and this is used to calculate the limit of detection (LOD) and quantification (LOQ) of the system (see Table 1 for definitions); above the linear range the signal will either plateau or start to decline. This is because, as described above, over a certain threshold the metal NPs used in SERS will be saturated and no more analyte will be within the LSP enhancement zone. In addition, if the aggregation conditions of the nanoparticles are not carefully controlled, large aggregates of NPs are formed that start to precipitate out of the colloid suspension also resulting in a decrease in response.

In order for peak areas to be reproducible then substrates with high fidelity and nanostructure uniformity must be generated and there are many factors when fabricating metal nanoparticles that need to be considered [21]. This is important and can be addressed with SERS substrates that are designed using rational means [22], or by exploiting statistical design of experiments (DOE) as demonstrated for the quantification of the illicit drug mephedrone [23], and the human $\beta$-blocker drug propranolol [24] as well as its quantification in human plasma, serum and urine using SERS [25].

Microfluidics can also be used to improve quantitative analysis with SERS as initially demonstrated by Keir et al. [12] who exploited these miniaturised devices for in situ nanoparticle production and aggregation. Popp and colleagues have also combined SERS with microfluidic devices as a general approach for quantitative analysis [26], which was recently demonstrated using the model analyte adenine [27].

Melamine is a substance that was added to milk to increase its nitrogen content and hence disguise the level of protein in watered down milk. This hazardous analyte caused the deaths of 6 infants in China and 300,000 victims in China alone were estimated to have been exposed to this poison, which was dispersed worldwide throughout the food supply chain [1]. It is perhaps unsurprising that given the importance of detecting melamine in milk, there have been several studies that have targeted SERS of this analyte [28-31]. Recently, $\mathrm{Hu}$ and coworkers developed molecularly imprinted polymers and reported an LOD and LOQ of 0.0165 and $0.055 \mathrm{mmol} / \mathrm{L}$ for the determination of melamine in milk [32, 33].

With respect to other food safety concerns, several potentially carcinogenic substances that may be present in food have also been targeted for quantitation with SERS and these include aflatoxins in maize [34], Sudan I spiked in chili powder [35] and Rhodamine B in chili oil [36]. Finally, although not hazardous to health the boar-taint compounds skatole and androstenone, which alter the flavor of meat, have also been recently detected and quantified in pork fat using SERS [37].

Finally, the detection of drugs of abuse is important and this has been shown using colloidal-based SERS for the quantification of the controlled substance tramadol [38] and the previously categorised legal high 5,6-methylenedioxy-2aminoindane (MDAI) [39]. SERS has also been used to measure drug overdoses 
from human saliva [40] as well as drugs of abuse [41, 42]. Clearly the detection and quantification of drugs is important and in contrast to the colloidal-based SERS used above this has also been possible using thin films produced via galvanic displacement [43], that have excellent reproducibility [44], as illustrated for the discrimination of a range of illicit materials [45].

\section{MULTIVARIATE ANALYSIS FOR QUANTIFICATION FROM SERS}

The univariate quantification detailed above works well when SERS peaks are present that are unique and are not crowded or overlapped with other spectral features. This may occur when an analyte is within a complex background (e.g. blood, urine or saliva) or when the goal is to perform quantitative multiplexed analysis of multiple analytes simultaneous. When this occurs more powerful algorithms are needed.

When univariate analysis fails it is prudent to use the whole spectrum, or a subset of the spectral features, as inputs to multivariate analysis (MVA) techniques. SERS data are by their nature multivariate and a typical spectrum from 200-3400 $\mathrm{cm}^{-1}$, at just $4 \mathrm{~cm}^{-1}$ resolution will contain 800 discrete descriptors. These descriptors are the columns in the spectra or termed 'bins'. Clearly the interpretation of such high dimensional data is difficult. Thus the underlying theme in MVA is simplification of the multivariate data into something that is over low dimension and hence more readily interpretable.

For quantitative analyses two general types of approaches are use in MVA: these may be exploratory analyses or multivariate regression based; these are introduced below, before some examples of their use for SERS are provided:

Exploratory data analyses are used to look at the relationship(s) between groups of samples. In these algorithms no prior information is required and so these are unsupervised in nature [46]. This means that the only information provided is the input data matrix. For SERS spectra this will be a group of $n$ samples by $m$ descriptors (bins), these are referred to as $X$-data. The most common unsupervised learning method is principal component analysis (PCA), others include dendrograms and (Kohonen) self-organising (feature) maps (SO(F)Ms).

PCA is a well-known and very old chemometrics approach $[47,48]$ that is used to reduce the dimensionality of multivariate data whilst preserving most of the variance. The results of PCA are a series of scores and loadings. The first score (PC1) is extracted to explain the most natural variance in the data and subsequent ones are extracted in decreasing order of importance [49]. Scores are used to inspect the relationship between samples and for quantitative analyses one would expect to see linkage in the scores ordinations plots which are related to quantification-related trends in the data; linkage in PCA is seen when points representing samples with different concentrations of a target analyte line up (i.e., are linked), and this pattern/trend reflects the increasing concentration of the target analyte. Inspection of the loadings plots allows the important spectral features to be discovered and for quantitative analysis this 
should resemble, to some degree, the pure SERS spectrum of the analyte that one is trying to quantify.

Not only do these exploratory multivariate approaches show quantitative trends in the data if they are present, but they can also be used to identify outliers. These outliers may fall outside the Hotelling $T^{2}$ 95\% confidence limit in PCA scores plots and this may be used to identify unusual samples that can arise from sample contamination or other analytical artifacts.

However unsupervised methods are descriptive in nature and require human interpretation. A much more powerful method of analysis is to use supervised learning methods that effect some multivariate regression (or mapping) of the SERS input data $(X$-data) onto the quantitative level of a target determinand $(Y$ data). The central feature of these methods is to ascertain whether SERS spectra can be correlated with a continuous variable (the quantitative level of a target analyte). Once calibrated, these methods allow quantitative prediction of unseen samples to this continuous variable. During calibration, a training set consisting of a series of training pairs is used, where each pair consists of a SERS spectrum ( $X$-data) with the concentration of an analyte ( $Y$-data) present with that spectrum. Usually a single analyte is investigated and the $Y$-data is a single $Y$ vector, however for multiplexing with, for example, 3 analytes to be measured simultaneous then for PLSR and ANNs the $Y$-data would be a matrix containing 3 columns; one for each of the analytes of interest.

Table 2 contains a summary of the most common multivariate chemometrics used for quantitative analysis, along with their key features. Partial least squares regression (PLSR) is a very common method which is largely used for linear regression [50]. Other methods such as support vector regression (SVR), which is a variant of support vector machines (SVMs) can in addition perform nonlinear mapping. A more in depth description of these algorithms and related chemometric methods are found here $[1,51]$.

Although multivariate analyses have been used in other fields of analytical chemistry, their application within SERS for quantitative analysis is relatively recent. Most applications of chemometrics to SERS are dominated by PLSR and these include the quantification of thymine [52], the quantitative detection of the spore biomarker dipicolinic acid (DPA) extracted from bacterial spores [53], quantitative assessments of the neurotransmitters glutamate and $\gamma$ aminobutyric acid in serum [54] and the accurate measurement of the fungicides thiram [55] and carbendazim [56] using SERS.

The above methods exploit machine learning in that the algorithm is first taught with samples of known provenance (i.e. concentration(s) of target analyte(s)), before its performance for quantification is tested with 'unknown' samples; these samples are unknown to the algorithm but known to the operator testing the stability of the system. An attractive alternative is to use chemometric algorithms that perform spectral unmixing/deconvolution. Multivariate curve resolution (MCR) is a method that is used to resolve mixtures by determining the number of individual components within a sample and is often combined with 
alternating least squares (ALS) for optimisation of the extracted component spectra [57]. This MCR-ALS approach generates two matrices: the first is a $C$ matrix which contains concentration profiles and along with it is the $S$ matrix which contains the corresponding pure spectra; which can be compared with the SERS spectra of pure analytes. For quantitative analysis from SERS spectra, MCRALS has been used to determine uric acid spiked into human urine at clinically relevant concentrations $\left(0.11 \mathrm{mmol} \mathrm{L}^{-1}\right)$ [58], and within the biocatalysis field for following the enzymatic turnover of substrates into products [59].

\section{MULTIPLEXED QUANTIFICATION FROM SERS OF MIXTURES}

SERS assays for quantification of specific DNA sequences allows for the analysis of low levels of DNA labeled with SERRS reporter molecules [60], and this approach has better analytical sensitivity than fluorescence based assays for the measurement of DNA [61]. For two recent reviews focusing on the quantification of DNA sequences using SERS the reader is directed here $[62,63]$.

One of the earliest examples of multiplex detection using SERS was reported by Faulds and colleagues who used two different excitation lasers for SERRS (to show the additional effect of resonance enhancements) and combined this with five different dyes in order to achieve quantification of 5-plex DNA-detection system with detection limits of $10^{-12} \mathrm{~mol} \mathrm{dm}^{3}$ [64]. This system did not use any chemometrics but relied on having five different SERS peaks being resolved.

Quantification of multiple dyes using SERS starts to get more complex when a single excitation source is used and so the same authors turned to multivariate analyses. In a study using six different dyes these authors were able to detect whether a labeled oligonucleotide was present or absent using PLSR in a total of $2^{6}$ (64 mixtures) [65], and this was also compared favourably with reanalysis of these data using Bayesian statistical methods [66]. More recently, Faulds and coworkers developed a novel quantitative assay for the simultaneous detection of three pathogens involved in meningitis using a combination of $\lambda$-exonuclease, SERRS and PLSR [67].

Detection of multiplex DNA sequences has also been reported using molecular beacon approaches using metal film over nanosphere (MFON) [68, 69] and silicon nanowire arrays (SiNWAr) with gold nanoparticles deposited onto the surface [70]. However both these approaches are negative assays that result in a decrease in SERS response upon target hybridisation and univariate analysis was used and quantification within the multiplex was not demonstrated.

The above assays use DNA hybridisation to detect and subsequently quantify a specific DNA sequence from a labeled reporter that is SERRS active. SERS from molecules within mixtures can also be developed for quantitative analysis of multiple analytes without recourse to chromatography. Alharbi et al. reported two studies investigating drugs and their metabolites. In the first study, ANNs outperformed PLSR in the quantification of the drug nicotine and its two major xenometabolites cotinine and trans-3'-hydroxycotinine [71]. This may have been 
because optimal aggregation and hence SERS signal was effected using 3 different $\mathrm{pH}$ conditions, rather than salts. Optimal SERS signals were seen at 3 different $\mathrm{pH}$ which corresponded to the 3 different $\mathrm{pKa}$ (acid dissociation constants) of the 3 analytes in the mixtures, and so all three spectra were concatenated and used in the ANN analyses. In a further study SERS with ANNs using 3 outputs ( $Y$-data) allowed for the quantification of caffeine and its major metabolites theobromine and paraxanthine [72], here a single $\mathrm{pH}$ environment was used for aggregation of the NPs and hence the chemometric analysis was simpler.

SERRS immunoassays have also previously been used for the specific detection of target antigen through selective antibody-antigen binding. A simple assay has been developed which utilises a SERRS labelled secondary detection antibody to detect directly the presence of the intracellular signalling protein p38 [73]. This demonstrated quantitative detection of a single target protein within an immunoassay; however, lower detection limits have been achieved by incorporating nanoparticles into the assay [74].

A SERS protein assay using gold nanoparticles functionalised with target antibodies and Raman reporter has been developed [75]. Nanoparticles were functionalised with a monolayer of bioselective detection antibodies by coupling through a strong Raman reporter molecule, DSNB (5,5 '-dithiobis(succinimidyl2-nitrobenzoate)). The functionalised nanoparticles were captured onto a gold coated glass slide through an antigen immunoassay leading to quantitative detection of CA 19-9 and MMP-7 using spatial multiplexing in an array format [76]. Methods to identify a number of analytes positively in situ have also been developed [77]. A protein immunoassay that utilises SERS detection to detect four target antigens simultaneously has been reported for the detection of multiple proteins using self-assembled mixed monolayer gold nanoparticles through a sandwich type immunoassay [78]. SERS has also been shown to outperform conventional ELISA assays for the detection of MUC4, expressed in pancreatic adenocarcinoma cell lines and tissues [79], and for the detection of p53 and p21 [80], as well hydrophilically-stabilised gold nanostars for the quantification of the tumour repressor p63 in cancerous tissues [81]. Multiplexed detection of PSA, thrombin and mucin-1 has also been achieved using aptamer functionalized nanoparticles [82] and a magnetic separation assay based on the use of paramagnetic nanoparticles for the detection of West Nile and Rift Valley fever virus [83].

Above are examples of where the detection of multiple analytes is possible within a mixture directly. However, when the mixture is very complex (e.g., from a patients blood or urine), or when there are multiple chemical species competing for the metal surface used for SERS, then alternative strategies are needed.

Liquid chromatography (LC) is one method that has been recently coupled with Raman and signals boosted using SERS (a combined method called LC-SERS). Cowcher and colleagues showed that using isocratic elution, where the mobile phase remained constant, silver colloid could be fed directly into the eluent 
stream from the LC. This subsequently underwent $\mathrm{KNO}_{3}$ mediated aggregation and was channelled through a quartz capillary for Raman/SERS interrogation. Targeted purine bases (adenine, guanine, hypoxanthine and xanthine) could be quantified with limits of detection (LoD) of 100-500 pmol [84]. This work is notable as it established for the first time that LC-SERS could be performed online; at the time other workers had only achieved off-line analysis by deposition of the eluent into wells containing SERS substrates (see for e.g. [85, 86]). More recently, an improved on-line LC-SERS system using reversed phase LC, where enhanced analyte separation was possible by programming the mobile phase during elution, showed that it was possible to target and quantitate methotrexate and its major metabolite 7-hydroxy methotrexate in urine from human patients suffering from rheumatoid arthritis [87].

On-line methods based on $\mathrm{Ag}$ (or $\mathrm{Au}$ ) colloids, while attractive, have the disadvantage that a continued supply of NPs is needed. By contrast, Schultz and colleagues have developed an attractive on-line system where the eluent from the LC is directed onto a SERS active surface using a sheath-flow interface that uses hydrodynamic focusing to confine analyte molecules eluting out of an LC column onto planar SERS substrates $[88,89]$. These authors have shown that it is possible to detect peptides and 20 amino acids separated by capillary electrophoresis [90,91] and to perform quantitative analysis of several metabolites [92].

\section{THE USE OF INTERNAL STANDARDS AND ISOTOPOLOGUES}

There are many factors that need to be considered when SERS is used for quantitative analysis. Three important factors are the number and degree of aggregation of the nanoparticles relative to the concentration of analytes within the interrogation voxel during analysis, how well the analyte binds to and orientates on the metal surface, and the power/fluence of the laser can vary (although we note that modern lasers do have improved stability). These are unfortunately inherently variable and hard to control with perfect precision, as small variations in either of these will alter the absolute signal output from SERS and hence the ability to perform accurate quantitative analysis.

One way to overcome these issues is to employ an internal standard (IS). One of the first examples of this was reported by Bell and colleagues who wanted to quantify dipicolinic acid (DPA; pyridine-2,6-dicarboxylic acid) [93]; DPA is important as it is a small molecule that is unique to Bacillus spores and so its detection and quantification can be an indication of potential anthrax attacks. However, in their study the SERS generated DPA signature from the symmetric ring stretch from pyridine evolved slowly over time due to the use of $\mathrm{Na}_{2} \mathrm{SO}_{4}$ as the aggregating agent. Therefore, to compensate for this temporal signal variation they used potassium thiocyanate as an internal standard and ratioed the pyridine to the CNS- vibration and this significantly improved quantification [93]. Following on from this, Cowcher and coworkers also quantified DPA from a nitric acid extract from Bacillus spores and used glutaric acid as the internal standard (KCNS was not used as it would have acid hydrolysed in these 
conditions producing CN!) [53]. Here the signal was also adjusted using a ratio calculation and they were able to quantify down to 7,000 spores $\left(10^{4}\right.$ spores are required for inhalation anthrax [93]). Finally, when Bell's group combined this with meso-droplets supported on superhydrophobic wires this allowed for the exquisitely sensitive detection of DPA which was equivalent to just 10 spores [94].

The problem with the use of these internal standards is there is still unequal competition between the target analyte and the internal standard (IS) on the metal surface. This means that even for similar chemical structures the signal may vary as a function of overall concentration and will not work above saturation of the NP surface [95]; this means the ratios of analyte to IS are not linear (as illustrated in [53]). One recent study has gone some way in overcoming this issue of competitive adsorption by placing the IS molecules within the inside of core-shell nanoparticles. A method that improved the quantitative analysis of target molecules over a large concentration range [96].

Isotopologues are one potential solution to the above problem and these are molecules that differ from each other only in their isotopic composition. For example, by the substitution with a stable isotope such as ${ }^{13} \mathrm{C}$ for ${ }^{12} \mathrm{C}$ or ${ }^{15} \mathrm{~N}$ for ${ }^{14} \mathrm{~N}$ or as illustrated in Figure 2 the substitution of $\mathrm{H}$ with $\mathrm{D}$. The substitution of hydrogen with deuterium is particularly attractive as the reduced mass of a functional group is very different as illustrated in the figure for the C-H to C-D substitution. This results in a shift in $\mathrm{CH}$ vibrations from $c a 2800 \mathrm{~cm}^{-1}$ to $c a 2100$ $\mathrm{cm}^{-1}$, and band ratios can then be made of the isotopologue to the natural isotope. This should have improved accuracy for quantification as the isotopic labelled molecules will compete in a very similar manner for the metal surface. Thereby the use of these internal standards will result in the reduction of any influences due to the number of nanoparticles within the analysis zone and fluctuations in laser fluence.

The use of isotopologues for SERS was first reported by van Duynes' group for systematic studies of single molecule detection of crystal violet [97]. For quantitative analysis, this approach is often termed isotope dilution surfaceenhanced Raman scattering (IDSERS) [98], and has been used to improve quantification between different batches of nanoparticles [99] and to effect the absolute quantification of markers in serum [100] as well as human plasma [101], and for the analysis of nicotine content in electronic cigarettes [102].

When competitive co-adsorption/co-association with the metal surface exists between different chemical species within complex matrices, (e.g.) in clinical samples, then isotopologues may not fully compensate for this. In these cases additional sample pre-treatment may be needed which would include selective (solvent) extraction of the target analyte. Here we would suggest the isotopologue is added before the extraction is undertaken to adjust for any variability in extraction efficiencies from such complex mixtures. 


\section{STANDARD ADDITION METHOD FOR ABSOLUTE QUANTIFICATION}

An alternative to using isotopologues or IDSERS is to use the standard addition method (SAM) for absolute quantification. The SAM is a well-established technique in analytical chemistry that can be used to effect absolute quantification of specific molecules within complex mixtures. Most SERS studies have quantified target analytes in simple matrices (e.g. water) and when more complex backgrounds are involved there can often be chemical species present which also compete for the metal NP surface needed for SERS and thus affect the ability to perform accurate quantifications.

As illustrated in Figure 3, SAM works by spiking known amounts of a standard into a sample of interest. This standard will be the molecule that one is trying to quantify. Plots of the peak area of a characteristic peak against the concentration of the standard spiked will yield a straight line. The equation of which is $\mathrm{y}=m \mathrm{x}+$ $b$; where $m$ and $b$ are the slope of the line and y-intercept, respectively. When the sample contains no analyte/standard the intercept will be 0 , by contrast when the sample already contains the analyte, then the intercept will be positive in the $y$-axis. From these plots (upper plot in Figure 3) the concentration of the analyte can be determined from the point at which the extrapolated line crosses the concentration axis ( $\mathrm{x}$ ) at zero signal (i.e., where $\mathrm{y}=0$ and thus $\mathrm{x}=-b / m$ such that the concentration $=b / m)[103,104]$.

In a series of elegant papers showing the evolution of lab-on-a-chip (LoC) SERS devices combined with SAM, Popp and colleagues developed the LOC device [105] after which they illustrated that using SAM improved quantification for the target analyte congo red [106], before going on to show that LoC with SAM allowed for the precise determination of the levels of the antibiotic nitroxoline spiked into human urine [107]. This group has also combined MCR-ALS with SAM for the quantification of nicotine in the presence of cotinine and anabasine in spikes of human urine using SERS [108]. Finally, other workers also exploited the SAM with SERS to analyse real-world samples when PLSR and MCR-ALS failed, and showed that this approach was able to effect absolute quantification of uric acid from urine from pregnant individuals suspected of having preeclampsia [109]. 


\section{CONCLUSIONS AND OUTLOOK}

This review has critiqued the development and application of SERS for quantitative analysis. Over the last five years there has been an explosion of interest in SERS for quantitative analysis including the analysis of multiple analytes simultaneously directly on the sample without recourse to any prior fractionation or chromatography. Much of this success has been due to improved chemometric analysis and the adoption of multivariate analyses, which are still quite new to this field, as well as the implementation of classic analytical chemistry methods borrowed from other fields. Most notable here is the use of isotopologues or IDSERS as internal standards and the exploitation of the standard addition method.

Recently the applications of SERS for quantitative analysis has moved out of simple chemistry based on mixture analysis in clean, uncomplicated backgrounds (viz. water), to more complex scenarios where accurate quantification of a variety of molecules has been possible in different human biofluids including blood plasma and serum as well as urine and saliva. We believe that SERS has evolved and is no longer an emerging technology. We predict that in the next decade this technique will be translated from the laboratory to the clinical environment and SERS will be exploited for quantitative analysis of many different chemical species. In addition, due to its inherent portability SERS will be an attractive point-of-care technology for at home testing [9] and for personalised medicine where it may replace more cumbersome and expensive analytical methods.

\section{ACKNOWLEDGEMENTS}

R.G. are also indebted to the UK BBSRC for continued funding of Raman spectroscopy and SERS (grants BB/K00199X/1; BB/G010250/1;

BB/L014823/1). DG and KF acknowledge support from the EPSRC healthcare technologies theme (EP/L014165/1).

\section{REFERENCES}

[1] D.I. Ellis, V.L. Brewster, W.B. Dunn, J.W. Allwood, A.P. Golovanov, R. Goodacre, Fingerprinting food: current technologies for the detection of food adulteration and contamination, Chemical Society Reviews, 41 (2012) 57065727.

[2] N.A. Refat, Z.S. Ibrahim, G.G. Moustafa, K.Q. Sakamoto, M. Ishizuka, S. Fujita, The induction of cytochrome P450 1A1 by Sudan Dyes, J. Biochem. Mol. Toxicol., 22 (2008) 77-84.

[3] A.D. Association, Standards of medical care in diabetes: Classification and diagnosis of diabetes, Diabetes Care, 40 (2017) S11-S24.

[4] A.G. Stephenson, L.S. LaPiana, D.R. Mulville, P.J. Rutledge, F.H. Bauer, D. Folta, G.A. Dukeman, R. Sackheim, P. Norvig, Mars Climate Orbiter Mishap Investigation Board Phase I Report, NASA, 1999. 
[5] A. Bonifacio, S.D. Marta, R. Spizzo, S. Cervo, A. Steffan, A. Colombatti, V. Sergo, Surface-enhanced Raman spectroscopy of blood plasma and serum using Ag and Au nanoparticles: a systematic study, Analytical and Bioanalytical Chemistry, 406 (2014) 2355-2365.

[6] W.B. Dunn, D. Broadhurst, H.J. Atherton, R. Goodacre, J.L. Griffin, Systems level studies of mammalian metabolomes: the roles of mass spectrometry and nuclear magnetic resonance spectroscopy, Chemical Society Reviews, 40 (2011) 387-426.

[7] W.B. Dunn, A. Erban, R.J.M. Weber, D.J. Creek, M. Brown, R. Brietling, T. Hankemeier, R. Goodacre, S. Neumann, J. Kopka, M.R. Viant, Mass Appeal: metabolite identification in mass spectrometry-focused untargeted metabolomics, Metabolomics, 9 (2013) S44-S66.

[8] D.I. Ellis, H. Muhamadali, S.A. Haughey, C.T. Elliott, R. Goodacre, Point-andshoot: rapid quantitative detection methods for on-site food fraud analysis moving out of the laboratory and into the food supply chain, Analytical Methods, 7 (2015) 9401-9414.

[9] D.K. Trivedi, K.A. Hollywood, R. Goodacre, Metabolomics for the masses: the future of metabolomics in a personalized world, New Horizons in Translational Medicine, 3 (2017) 294-305.

[10] M. Fleischmann, P.J. Hendra, A.J. McQuillan, Raman spectra of pyridine adsorbed at a silver electrode, Chemical Physics Letters, 26 (1974) 163-166.

[11] D. Graham, R. Goodacre, Graham, D. \& Goodacre, R. (2008) Chemical and bioanalytical applications of surface enhanced Raman scattering spectroscopy. Chemical Society Reviews 37, 883-884, Chemical Society Reviews, 37 (2008) 883-884.

[12] R. Keir, E. Igata, M. Arundell, W.E. Smith, D. Graham, C. McHugh, J.M. Cooper, SERRS. In Situ Substrate Formation and Improved Detection Using Microfluidics, Analytical Chemistry, 74 (2002) 1503-1508.

[13] S. Nie, S.R. Emory, Probing single molecules and single nanoparticles by surface-enhanced Raman scattering, Science, 275 (1997) 1102-1106.

[14] E.J. Blackie, E.C. Le Ru, P.G. Etchegoin, Single-molecule surface-enhanced Raman spectroscopy of nonresonant molecules, Journal of the American Chemical Society, 131 (2009) 14466-14472.

[15] D.L. Jeanmarie, R.P. Van Duyne, Surface raman spectroelectrochemistry: Part I. Heterocyclic, aromatic, and aliphatic amines adsorbed on the anodized silver electrode, Journal of Electroanalytical Chemistry and Interfacial Electrochemistry, 84 (1977) 1-10.

[16] M. Moskovits, Surface-Enhanced Spectroscopy, Rev. Mod. Phys., 57 (1985) 783-826.

[17] M.G. Albrecht, J.A. Creighton, Anomalously intense Raman spectra of pyridine at a silver electrode, Journal of the American Chemical Society 99 (1977) 5215-5217.

[18] C. May, X.-S. Zheng, K. Weber, J. Popp, Recent progress in surface-enhanced Raman spectroscopy for biological and biomedical applications: from cells to clinics, Chemical Society Reviews, 13 (2017) 3945-3961.

[19] M.J. Pelletier, Quantitative analysis using Raman spectrometry, Applied Spectroscopy, 57 (2003) 20A-42A. 
[20] K. Faulds, W.E. Smith, D. Graham, Evaluation of surface-enhanced resonance Raman scattering for quantitative DNA analysis, Analytical Chemistry, 76 (2004) 412-417.

[21] H. Fisk, C. Westley, N.J. Turner, R. Goodacre, Achieving optimal SERS through enhanced experimental design, Journal of Raman Spectroscopy, 47 (2016) 59-66.

[22] Y.L. Wang, S. Schlucker, Rational design and synthesis of SERS labels, Analyst, 138 (2013) 2224-2238.

[23] S. Mabbott, E. Correa, D.P. Cowcher, J.W. Allwood, R. Goodacre, Optimization of Parameters for the Quantitative Surface-Enhanced Raman Scattering Detection of Mephedrone Using a Fractional Factorial Design and a Portable Raman Spectrometer, Analytical Chemistry, 85 (2013) 923-931.

[24] C. Levene, E. Correa, E.W. Blanch, R. Goodacre, Enhancing surface enhanced Raman scattering (SERS) detection of Propranolol with multiobjective evolutionary optimization, Analytical Chemistry, 84 (2012) 7899-7905.

[25] A. Subaihi, L. Almanqur, H. Muhamadali, N. AlMasoud, D.I. Ellis, D.K. Trivedi, K.A. Hollywood, Y. Xu, R. Goodacre, Rapid, Accurate, and Quantitative Detection of Propranolol in Multiple Human Biofluids via Surface-Enhanced Raman Scattering, Analytical Chemistry, 88 (2016) 10884-10892.

[26] A. Marz, B. Monch, A. Walter, T. Bocklitz, W. Schuhmacher, P. Rosch, M. Kiehntopf, J. Popp, The multifunctional application of microfluidic lab-on-achip surface enhanced Raman spectroscopy (LOC-SERS) within the field of bioanalytics, in: N. Ramanujam, J. Popp (Eds.) Clinical and Biomedical Spectroscopy and Imaging Ii2011.

[27] E. Kammer, K. Olschewski, T. Bocklitz, P. Rosch, K. Weber, D. Cialla, J. Popp, A new calibration concept for a reproducible quantitative detection based on SERS measurements in a microfluidic device demonstrated on the model analyte adenine, Physical Chemistry Chemical Physics, 16 (2014) 90569063.

[28] X.F. Zhang, M.Q. Zou, X.H. Qi, F. Liu, X.H. Zhu, B.H. Zhao, Detection of melamine in liquid milk using surface-enhanced Raman scattering spectroscopy, Journal of Raman Spectroscopy, 41 (2010) 1655-1660.

[29] T.T. Lou, Y.Q. Wang, J.H. Li, H.L. Peng, H. Xiong, L.X. Chen, Rapid detection of melamine with 4-mercaptopyridine-modified gold nanoparticles by surfaceenhanced Raman scattering, Analytical and Bioanalytical Chemistry, 401 (2011) 333-338.

[30] N.N. Yazgan, I.H. Boyaci, A. Topcu, U. Tamer, Detection of melamine in milk by surface-enhanced Raman spectroscopy coupled with magnetic and Raman-labeled nanoparticles, Analytical and Bioanalytical Chemistry, 403 (2012) 2009-2017.

[31] T.T. Lang, S. Pang, L.L. He, Integration of colorimetric and SERS detection for rapid screening and validation of melamine in milk, Analytical Methods, 7 (2015) 6426-6431.

[32] Y.X. Hu, S.L. Feng, F. Gao, E.C.Y. Li-Chan, E. Grant, X.N. Lu, Detection of melamine in milk using molecularly imprinted polymers-surface enhanced Raman spectroscopy, Food Chemistry, 176 (2015) 123-129.

[33] Y.X. Hu, X.N. Lu, Rapid Detection of Melamine in Tap Water and Milk Using Conjugated "One-Step" Molecularly Imprinted Polymers-Surface Enhanced 
Raman Spectroscopic Sensor, Journal of Food Science, 81 (2016) N1272N1280.

[34] K.M. Lee, T.J. Herrman, Y. Bisrat, S.C. Murray, Feasibility of Surface-Enhanced Raman Spectroscopy for Rapid Detection of Aflatoxins in Maize, Journal of Agricultural and Food Chemistry, 62 (2014) 4466-4474.

[35] W. Cheung, I.T. Shadi, Y. Xu, R. Goodacre, Quantitative Analysis of the Banned Food Dye Sudan-1 Using Surface Enhanced Raman Scattering with Multivariate Chemometrics, Journal of Physical Chemistry C, 114 (2010) 7285-7290.

[36] C. Wang, F.S. Cheng, Y.H. Wang, Z.J. Gong, M.K. Fan, J.M. Hu, Single point calibration for semi-quantitative screening based on an internal reference in thin layer chromatography-SERS: the case of Rhodamine B in chili oil, Analytical Methods, 6 (2014) 7218-7223.

[37] K.M. Sorensen, C. Westley, R. Goodacre, S.B. Engelsen, Simultaneous quantification of the boar-taint compounds skatole and androstenone by surface-enhanced Raman scattering (SERS) and multivariate data analysis, Analytical and Bioanalytical Chemistry, 407 (2015) 7787-7795.

[38] O. Alharbi, Y. Xu, R. Goodacre, Detection and quantification of the opioid tramadol in urine using surface enhanced Raman scattering, Analyst, 140 (2015) 5965-5970.

[39] S. Mabbott, O. Alharbi, K. Groves, R. Goodacre, Application of surface enhanced Raman scattering to the solution based detection of a popular legal high, 5,6-methylenedioxy-2-aminoindane (MDAI), Analyst, 140 (2015) 43994406.

[40] S. Farquharson, C. Shende, A. Sengupta, H. Huang, F. Inscore, Rapid Detection and Identification of Overdose Drugs in Saliva by Surface-Enhanced Raman Scattering Using Fused Gold Colloids, Pharmaceutics, 3 (2011) 425-439.

[41] C. Andreou, M.R. Hoonejani, M.R. Barmi, M. Moskovits, C.D. Meinhart, Rapid Detection of Drugs of Abuse in Saliva Using Surface Enhanced Raman Spectroscopy and Microfluidics, ACS Nano, 7 (2013) 7157-7164.

[42] K. Faulds, W.E. Smith, D. Graham, R.J. Lacey, Assessment of silver and gold substrates for the detection of amphetamine sulfate by surface enhanced Raman scattering (SERS), Analyst, 127 (2002) 282-286.

[43] S. Mabbott, I.A. Larmour, V. Vishnyakov, Y. Xu, D. Graham, R. Goodacre, The optimisation of facile substrates for surface enhanced Raman scattering through galvanic replacement of silver onto copper, Analyst, 137 (2012) 2791-2798.

[44] S. Mabbott, Y. Xu, R. Goodacre, Objective assessment of SERS thin films: comparison of silver on copper via galvanic displacement with commercially available fabricated substrates, Analytical Methods, 9 (2017) 4783-4789.

[45] S. Mabbott, A. Eckmann, C. Casiraghi, R. Goodacre, 2p or not 2p: tuppencebased SERS for the detection of illicit materials, Analyst, 138 (2013) 118122.

[46] R.G. Brereton, Chemometrics: Data Analysis for the Laboratory and Chemical Plant, John Wiley \& Sons, Ltd2003.

[47] K. Pearson, On lines and planes of closest fit to systems of points in space, Philosophical Magazine, 2 (1901) 559-572. 
[48] H. Hotelling, Analysis of a complex of statistical variables into principal components, Journal of Educational Psychology, 24 (1933) 417-441, 498520.

[49] I.T. Jolliffe, Principal Component Analysis, Springer-Verlag, New York, 1986.

[50] H. Martens, T. Næs, Multivariate Calibration, John Wiley, Chichester, 1989.

[51] P.S. Gromski, H. Muhamadali, D.I. Ellis, Y. Xu, E. Correa, M.L. Turner, R. Goodacre, A tutorial review: Metabolomics and partial least squaresdiscriminant analysis - a marriage of convenience or a shotgun wedding, Analytica Chimica Acta, 879 (2015) 10-23.

[52] L. Zhang, Q. Li, W. Tao, B. Yu, Y. Du, Quantitative analysis of thymine with surface-enhanced Raman spectroscopy and partial least squares (PLS) regression, Analytical and Bioanalytical Chemistry, 398 (2010) 1827-1832.

[53] D.P. Cowcher, Y. Xu, R. Goodacre, Portable, quantitative detection of Bacillus bacterial spores using surface-enhanced Raman scattering, Analytical Chemistry, 85 (2013) 3297-3302.

[54] A.M.T. Monfared, T. V.S., T. V.L., H. Anis, Surface-enhanced Raman scattering spectroscopy for the detection of glutamate and gamma-aminobutyric acid in serum by partial least squares analysis, IEEE Photonics Journal, 7 (2015) 3900216.

[55] W. Shizhuang, C. Sheng, L. Miao, Z. Xinhua, Z. Shouguo, Z. Jian, C. Chen Jin, C. Lei, Quantitative analysis of thiram based on SERS and PLSR combined with wavenumber selection, Analytical Methods, 6 (2014) 242-247.

[56] A.D. Strickland, C.A. Batt, Detection of carbendazim by surface-enhanced raman scattering using cyclodextrin inclusion complexes on gold nanorods, Analytical Chemistry, DOI (2017) in press.

[57] R. Tauler, Multivariate curve resolution applied to second order data, Chemometrics and Intelligent Laboratory Systems, 30 (1995) 133-146.

[58] J.E.L. Villa, R.J. Poppi, A portable SERS method for the determination of uric acid using a paper-based substrate and multivariate curve resolution, Analyst, 141 (2016) 1966-1972.

[59] C. Westley, Y. Xu, A.J. Carnell, N.J. Turner, R. Goodacre, Label-free surface enhanced Raman scattering approach for high-throughput screening of biocatalysts, Analytical Chemistry, 88 (2016) 5898-5903.

[60] D. Graham, K. Faulds, Quantitative SERRS for DNA sequence analysis, Chemical Society Reviews, 37 (2008) 1042-1051.

[61] K. Faulds, R.P. Barbagallo, J.T. Keer, W.E. Smith, D. Graham, SERRS as a more sensitive technique for the detection of labelled oligonucleotides compared to fluorescence, Analyst, 129 (2004) 567-568.

[62] J.A. Dougan, K. Faulds, Surface enhanced Raman scattering for multiplexed detection, Analyst, 137 (2012) 345-554.

[63] S. Laing, K. Gracie, K. Faulds, Multiplex in vitro detection using SERS, Chemical Society Reviews, 45 (2016) 1901-1918.

[64] K. Faulds, F. McKenzie, W.E. Smith, D. Graham, Quantitative simultaneous multianalyte detection of DNA by dual - wavelength surface - enhanced resonance Raman scattering, Angewandte Chemie, 119 (2007) 1861-1863.

[65] K. Faulds, R.M. Jarvis, W.E. Smith, D. Graham, R. Goodacre, Multiplexed detection of labelled oligonucleotides using surface enhanced resonance Raman scattering (SERRS), Analyst, 133 (2008) 1505-1512. 
[66] M. Zhong, M. Girolami, K. Faulds, D. Graham, Bayesian methods to detect dye - labelled DNA oligonucleotides in multiplexed Raman spectra, Journal of the Royal Statistical Society: Series C (Applied Statistics), 60 (2011) 187206.

[67] K. Gracie, E. Correa, S. Mabbott, J.A. Dougan, D. Graham, R. Goodacre, K. Faulds, Simultaneous detection and quantification of three bacterial meningitis pathogens by SERS, Chemical Science, 5 (2014) 1030-1040.

[68] H.T. Ngo, H.-N. Wang, A.M. Fales, T. Vo-Dinh, Label-free DNA biosensor based on SERS molecular sentinel on nanowave chip, Analytical Chemistry, 85 (2013) 6378-6383.

[69] H.T. Ngo, H.-N. Wang, T. Burker, G.S. Ginsburg, T. Vo-Dinh, Multiplex detection of disease biomarkers using SERS molecular sentinel-on-chip, Analytical and Bioanalytical Chemistry, 406 (2014) 335-3344.

[70] Y. Guo, X. Jiang, Y. Zhong, Y. Su, C. Fan, S.-T. Lee, Y. He A molecular beaconbased signal-off surface-enhanced Raman scattering strategy for highly sensitive, reproducible, and multiplexed DNA detection, Small, 9 (2013) 2493-2499.

[71] O. Alharbi, Y. Xu, R. Goodacre, Simultaneous multiplexed quantification of nicotine and its metabolites using surface enhanced Raman scattering, Analyst, 139 (2014) 4820-4827.

[72] O. Alharbi, Y. Xu, R. Goodacre, Simultaneous multiplexed quantification of caffeine and its major metabolites theobromine and paraxanthine using surface-enhanced Raman scattering, Analytical and Bioanalytical Chemistry, 407 (2015) 8253-8261.

[73] P. Douglas, R.J. Stokes, D. Graham, W.E. Smith, Immunoassay for P38 MAPK using surface enhanced resonance Raman spectroscopy (SERRS), Analyst, 133 (2008) 791-796.

[74] J.D. Driskell, J.M. Uhlenkamp, R.J. Lipert, M.D. Porter, Surface-enhanced Raman scattering immunoassays using a rotated capture substrate, Analytical Chemistry, 79 (2007) 4141-4148.

[75] D.S. Grubisha, R.J. Lipert, H.Y. Park, J.D. Driskell, M.D. Porter, Femtomolar detection of prostate-specific antigen: an immunoassay based on surfaceenhanced Raman scattering and immunogold labels, Analytical Chemistry, 75 (2003) 5936-5943.

[76] J.H. Granger, M.C. Granger, M.A. Firpo, S.J. Mulvihill, M.D. Porter, Toward development of a surface-enhanced Raman scattering (SERS)-based cancer diagnostic immunoassay panel, Analyst, 138 (2013) 410-416.

[77] X.X. Han, L. Chen, J. Guo, B. Zhao, Y. Ozaki, Coomassie brilliant dyes as surface-enhanced Raman scattering probes for protein-ligand recognitions, Analytical Chemistry, 82 (2010) 4102-4106.

[78] G. Wang, H.Y. Park, R.J. Lipert, M.D. Porter, Mixed monolayers on gold nanoparticle labels for multiplexed surface-enhanced Raman scattering based immunoassays, Analytical Chemistry, 81 (2009) 9643-9650.

[79] G. Wang, R.J. Lipert, M. Jain, S. Kaur, S. Chakraboty, M.P. Torres, S.K. Batra, R.E. Brand, M.D. Porter, Detection of the potential pancreatic cancer marker MUC4 in serum using surface-enhanced Raman scattering, Analytical Chemistry, 83 (2011) 2554-2561. 
[80] L. Wu, Z. Wang, S. Zong, H. Chen, C. Wang, S. Xua, Y. Yiping Cui, Simultaneous evaluation of p53 and p21 expression level for early cancer diagnosis using SERS technique, Analyst, 138 (2013) 3450-3456.

[81] M. Schütz, D. Steinigeweg, M. Salehi, K. Kömpe, S. Schlücker, Hydrophilically stabilized gold nanostars as SERS labels for tissue imaging of the tumor suppressor p63 by immuno-SERS microscopy, Chemical Communications, 47 (2011) 4216-4218.

[82] L. Xu, W. Yan, W. Ma, H. Kuang, X. Wu, L. Liu, Y. Zhao, L. Wang, C. Xu, SERS encoded silver pyramids for attomolar detection of multiplexed disease biomarkers, Advanced Materials, 27 (2015) 1706-1711.

[83] J. Neng, M.H. Harpster, W.C. C.Wilson, P.A. Johnson, Surface-enhanced Raman scattering (SERS) detection of multiple viral antigens using magnetic capture of SERS-active nanoparticles, Biosensors and Bioelectronics, 41 (2013) 316321.

[84] D.P. Cowcher, R. Jarvis, R. Goodacre, Quantitative online liquid chromatography-surface-enhanced Raman scattering of purine bases, Analytical Chemistry, 86 (2014) 9977-9984.

[85] R.M. Seifar, R.J. Dijkstra, U.A.T. Brinkman, C. Gooijer, At-line coupling of surface-enhanced resonance Raman spectroscopy and reversed-phase ionpair chromatography, Analytical Communications, 36 (1999) 273-276.

[86] G. Trachta, B. Schwarze, B. Sagmuller, G. Brehm, S. Schneider, Combination of high-performance liquid chromatography and SERS detection applied to the analysis of drugs in human blood and urine, Journal of Molecular Structure, 693 (2004) 175-185.

[87] A. Subaihi, D.K. Trivedi, K.A. Hollywood, J. Bluett, Y. Xu, H. Muhamadali, D.I. Ellis, R. Goodacre, Quantitative Online Liquid Chromatography SurfaceEnhanced Raman Scattering (LC-SERS) of Methotrexate and its Major Metabolites, Analytical Chemistry, 89 (2017) 6702-6709.

[88] M.R. Bailey, A.M. Pentecost, A. Selimovic, R.S. Martin, Z.D. Schultz, Sheathflow microfluidic approach for combined surface enhanced Raman scattering and electrochemical detection, Analytical Chemistry, 87 (2015) 4347-4355.

[89] C.M. Riordan, K.T. Jacobs, P. Negri, Z.D. Schultz, Sheath flow SERS for chemical profiling in urine, Faraday Discussions, 187 (2016) 473-484.

[90] P. Negri, Z.D. Schultz, Online SERS detection of the 20 proteinogenic L-amino acids separated by capillary zone electrophoresis, Analyst, 139 (2014) 59895998.

[91] P. Negri, S.A. Sarver, N.M. Schiavone, N.J. Dovichi, Z.D. Schultz, Online SERS detection and characterization of eight biologically-active peptides separated by capillary zone electrophoresis, Analyst, 140 (2015) 1516-1522.

[92] A. Nguyen, Z.D. Schultz, Quantitative online sheath-flow surface enhanced Raman spectroscopy detection for liquid chromatography, Analyst, 141 (2016) 3630-3635.

[93] S.E.J. Bell, J.N. Mackle, N.M.S. Sirimuthu, Quantitative surface-enhanced Raman spectroscopy of dipicolinic acid - towards rapid anthrax endospore detection, Analyst, 130 (2005) 545-549.

[94] M. Cheung, W.W.Y. Lee, D.P. Cowcher, R. Goodacre, S.E.J. Bell, SERS of mesodroplets supported on superhydrophobic wires allows exquisitely sensitive 
detection of dipicolinic acid, an anthrax biomarker, considerably below the infective close, Chemical Communications, 52 (2016) 9925-9928.

[95] A. Stewart, S. Zheng, M.R. McCourt, S.E.J. Bell, Controlling assembly of mixed thiol monolayers on silver nanoparticles to tune their surface properties, ACS Nano, 6 (2012) 3718-3726.

[96] W.L. Shen, X., C. Jiang, C. Li, H. Lin, J. Huang, S. Wang, G. Liu, X. Yan, Q. Zhong, B. Ren, Reliable quantitative SERS analysis facilitated by core-shell nanoparticles with embedded internal standards, Angewandte Chemie, 54 (2015) 7308-7312.

[97] S.L. Kleinman, E. Ringe, N. Valley, K.L. Wustholz, E. Phillips, K.A. Scheidt, G.C. Schatz, R.P. Van Duyne, Single-molecule surface-enhanced Raman spectroscopy of crystal violet isotopologues: theory and experiment, Journal of the American Chemical Society, 133 (2011) 4115-4122.

[98] S. Zakel, S. Wundrack, G. O'Connor, B. Güttler, R. Stosch, Validation of isotope dilution surface-enhanced Raman scattering (IDSERS) as a higher order reference method for clinical measurands employing international comparison schemes, Journal of Raman Spectroscopy, 44 (2013) 1246-1252.

[99] A. Subaihi, Y. Xu, H. Muhamadali, S.T. Mutter, E.W. Blanch, D.I. Ellis, R. Goodacre, Towards improved quantitative analysis using surface-enhanced Raman scattering incorporating internal isotope labelling, Analytical Methods, DOI (2017) in press.

[100] S. Zakel, O. Rienitz, B. Güttler, R. Stosch, Double isotope dilution surfaceenhanced Raman scattering as a reference procedure for the quantification of biomarkers in human serum, Analyst, 136 (2011) 3956-3961.

[101] A. Subaihi, H. Muhamadali, S.T. Mutter, E. Blanch, D.I. Ellis, R. Goodacre, Quantitative detection of codeine in human plasma using surface-enhanced Raman scattering via adaptation of the isotopic labelling principle, Analyst, 142 (2017) 1099-1105.

[102] N. Itoh, S.E.J. Bell, High dilution surface-enhanced Raman spectroscopy for rapid determination of nicotine in e-liquids for electronic cigarettes, Analyst, 142 (2017) 994-998.

[103] G.R. Bruce, P.S. Gill, Estimates of precision in a standard additions analysis, Journal of Chemical Education, 76 (1999) 805-807.

[104] T. Stark, N. Wollmann, S. Losch, T. Hofmann, Quantitation of resveratrol in red wines by means of stable isotope dilution analysis-ultra-performance liquid chromatography-quan-time-of-flight mass spectrometry and cross validation, Analytical Chemistry, 83 (2011) 3398-3405.

[105] I.J. Hidi, A. Muhlig, M. Jahn, F. Liebold, D. Cialla, K. Weber, J. Popp, LOCSERS: towards point-of-care diagnostic of methotrexate, Analytical Methods, 6 (2014) 3943-3947.

[106] E. Kammer, K. Olschewski, S. Stockel, P. Rosch, K. Weber, D. Cialla-May, T. Bocklitz, J. Popp, Quantitative SERS studies by combining LOC-SERS with the standard addition method, Analytical and Bioanalytical Chemistry, 407 (2015) 8925-8929.

[107] I.J. Hidi, M. Jahn, K. Weber, T. Bocklitz, M.W. Pletz, D. Cialla-May, J. Popp, Lab-on-a-chip-surface enhanced Raman scattering combined with the standard addition method: toward the quantification of nitroxoline in spiked human urine samples, Analytical Chemistry, 88 (2016) 9173-9180. 
[108] M.B. Mamian-Lopez, R.J. Poppi, Standard addition method applied to the urinary quantification of nicotine in the presence of cotinine and anabasine using surface enhanced Raman spectroscopy and multivariate curve resolution, Analytica Chimica Acta, 760 (2013) 53-59.

[109] C. Westley, Y. Xu, B. Thilaganathan, A.J. Carnell, N.J. Turner, R. Goodacre, Absolute quantification of uric acid in human urine using surface enhanced Raman scattering with the standard addition method, Analytical Chemistry, 89 (2017) 2472-2477.

[110] J.D. Womack, T.J. Vickers, C.K. Mann, Determination of azo dyes by resonance-enhanced Raman spectroscopy, Applied Spectroscopy, 41 (1987) 117-119. 


\section{TABLES}

Table 1. Terms and criteria used to assess a model's ability to quantify a target determinand accurately

\begin{tabular}{|c|c|c|}
\hline Term & Type of analysis & Use/Definition \\
\hline LOD & Statistics based & $\begin{array}{l}\text { Limit of Detection: } 3 \times \text { the standard deviation of } \\
\text { the blank }{ }^{\S}\end{array}$ \\
\hline LOQ & Statistics based & $\begin{array}{l}\text { Limit of Quantification: } 10 \times \text { the standard } \\
\text { deviation of the blank }\end{array}$ \\
\hline$R^{2}$ & $\begin{array}{l}\text { Correlation } \\
\text { coefficient }\end{array}$ & $\begin{array}{l}\text { Assesses linearity in prediction from data used to } \\
\text { train the model }\end{array}$ \\
\hline$Q^{2}$ & $\begin{array}{l}\text { Correlation } \\
\text { coefficient }\end{array}$ & $\begin{array}{l}\text { Assesses linearity in prediction from data used to } \\
\text { test the model }\end{array}$ \\
\hline RMSEC & Error based & $\begin{array}{l}\text { Root mean squared error (RMSE) in the } \\
\text { calibration (C) data used to train the model }\end{array}$ \\
\hline RMSECV & Error based & $\begin{array}{l}\text { RMSE in the cross-validation (CV) data used } \\
\text { during the calibration of the model to set certain } \\
\text { internal parameters* }\end{array}$ \\
\hline RMSEP & Error based & $\begin{array}{l}\text { The prediction (P) RMSE of the independent test } \\
\text { set data not used during calibration }\end{array}$ \\
\hline
\end{tabular}

$\S \quad$ The lowest amount of a substance detected for a specific peak. Defined as the detectable signal greater than the blank sample, with a $1 \%$ confidence limit [110].

* For PLSR this will be the number of latent variables (LVs) to use 
Table 2 Common multivariate chemometric methods used for quantification of target analytes

\begin{tabular}{|c|c|c|}
\hline Abb. & Algorithm & Key features \\
\hline CCA & $\begin{array}{l}\text { Canonical } \\
\text { correlation } \\
\text { analysis }\end{array}$ & $\begin{array}{l}\text { - } \text { Assesses the relationship between two sets of } \\
\text { variables } X \text { and } Y \\
\text { - Latent variables (LVs) inform which features are } \\
\text { correlated } \\
\text { - Performs a linear mapping from } X \text { to } Y\end{array}$ \\
\hline MLR & $\begin{array}{l}\text { Multivariate } \\
\text { linear } \\
\text { regression }\end{array}$ & $\begin{array}{l}\text { - Performs simple regression analysis on multiple } \\
\text { input variables ( } X \text {-data) } \\
\text { - Performs a linear mapping from } X \text { to } Y\end{array}$ \\
\hline PCR & $\begin{array}{l}\text { Principal } \\
\text { components } \\
\text { regression }\end{array}$ & $\begin{array}{l}\text { - Uses principal components analysis on } X \text {-data } \\
\text { - Extracted PC are regressed against the target }(Y) \\
\text { - Performs a linear mapping from } X \text { to } Y\end{array}$ \\
\hline PLSR & $\begin{array}{l}\text { Partial least } \\
\text { squares } \\
\text { regression }\end{array}$ & $\begin{array}{l}\text { - Performs simultaneous regression on } X \text { and } Y \\
\text { - Can be used to predict multiple variables (PLS2) } \\
\text { - Performs a linear mapping from } X \text { to } Y\end{array}$ \\
\hline SVR & $\begin{array}{l}\text { Support } \\
\text { vector } \\
\text { regression }\end{array}$ & $\begin{array}{l}\text { - Can use many potential kernel functions } \\
\text { - Distribution of samples does not influence output } \\
\text { - Performs both linear and non-linear mapping } \\
\text { (dependent on kernel) }\end{array}$ \\
\hline ANN & $\begin{array}{l}\text { Artificial } \\
\text { neural } \\
\text { network }\end{array}$ & $\begin{array}{l}\text { - Machine learning technique based on learning } \\
\text { - Many different learning approaches } \\
\text { - Converges to answer relatively slowly (compared to } \\
\text { the other techniques in this table) } \\
\text { - Very powerful non-linear mapping technique }\end{array}$ \\
\hline MCR & $\begin{array}{l}\text { Multivariate } \\
\text { curve } \\
\text { resolution }\end{array}$ & $\begin{array}{l}\text { - Method used to resolve mixtures by determining the } \\
\text { number of individual components within a sample } \\
\text { - Generates two matrices: } C=\text { concentration profiles } \\
\text { and } S=\text { corresponding pure spectra } \\
\text { - Often combined with alternating least squares (ALS) } \\
\text { for optimisation of the extracted component spectra }\end{array}$ \\
\hline
\end{tabular}




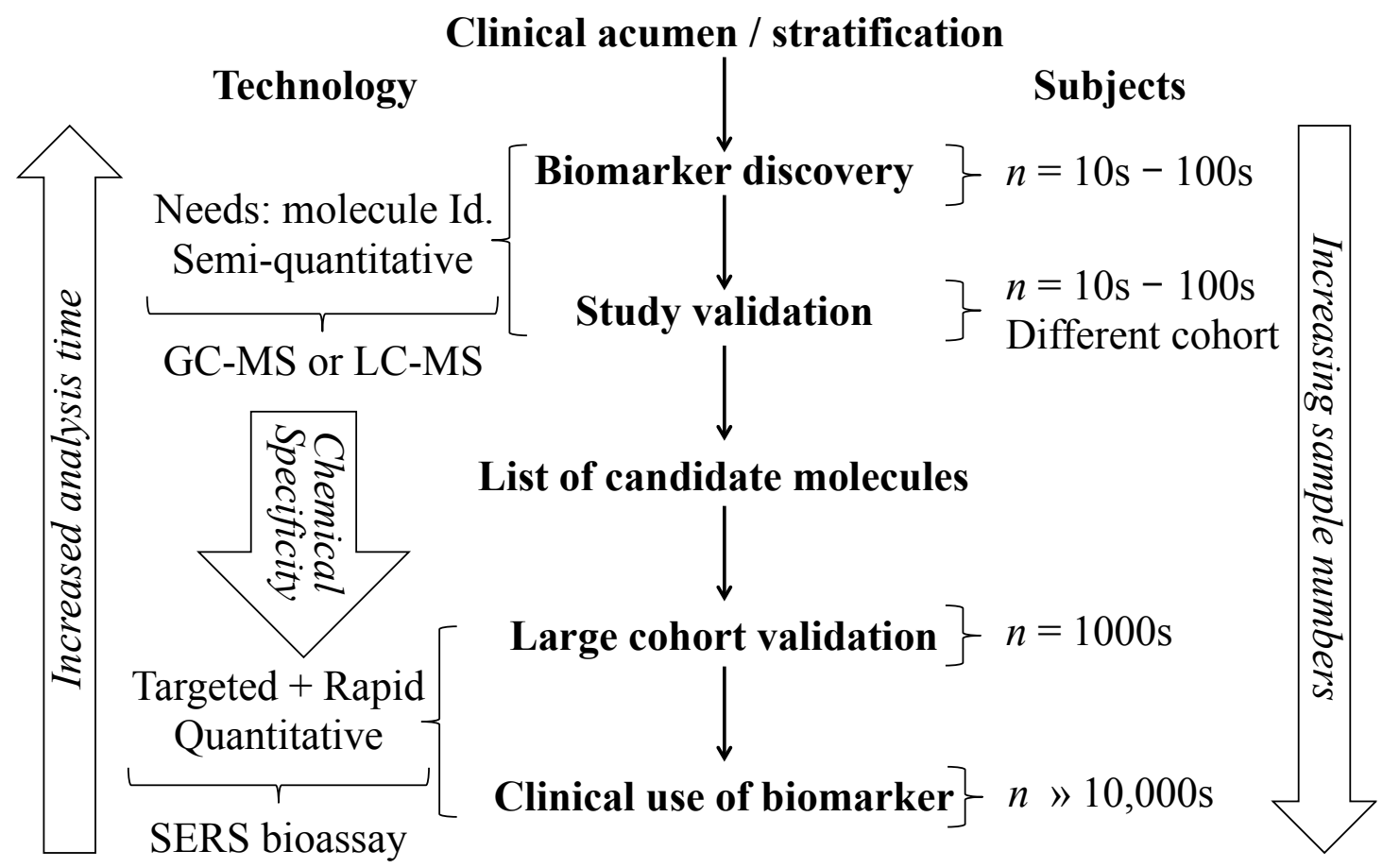

Figure 1 The biomarker discovery pipeline. This starts with the discovery of a new biomarker - DNA, RNA, protein, metabolite - that allows stratification of patients into (e.g.) patients with disease from healthy matched controls. Once candidate biomarkers are discovered then higher throughput and chemical specific analyses based on SERS bioassays are used for epidemiological assessment before delivery of the new biomarker into the clinic for use. 

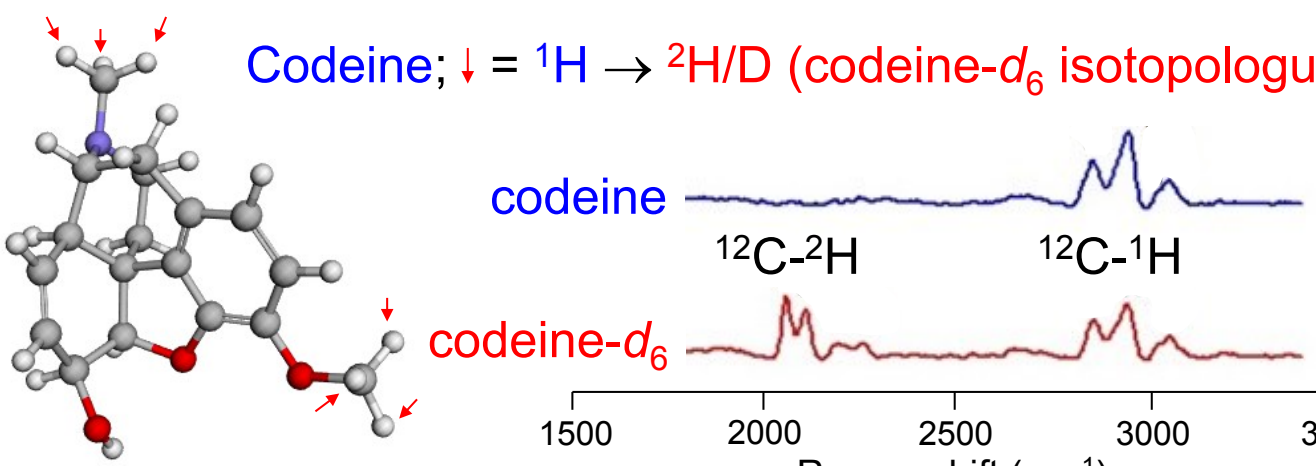

codeine- $d_{6}$

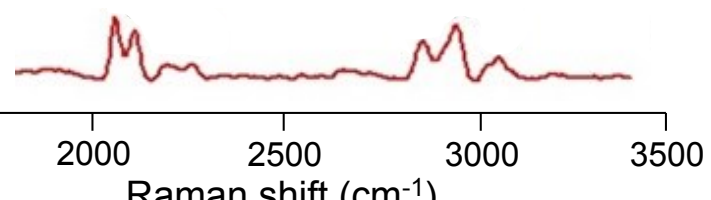

$$
v=\frac{1}{2 \pi} \sqrt{\frac{k}{\mu}} \longrightarrow \mu=\frac{m_{1} m_{2}}{m_{1}+m_{2}}
$$

$$
\text { Where: } \begin{aligned}
v & =\text { vibrational frequency } & \therefore & { }^{12} \mathrm{C}-{ }^{-} \mathrm{H}, \mu=12 / 13=0.92 \\
k & =\text { force constant } & & { }^{13} \mathrm{C}-{ }^{-} \mathrm{H}, \mu=13 / 14=0.93 \\
\mu & =\text { reduced mass } & & { }^{12} \mathrm{C}-{ }^{2} \mathrm{H}, \mu=24 / 14=1.71
\end{aligned}
$$

Figure 2 The use of isotopologues for absolute quantification using codeine- $d_{6}$ as an example. 


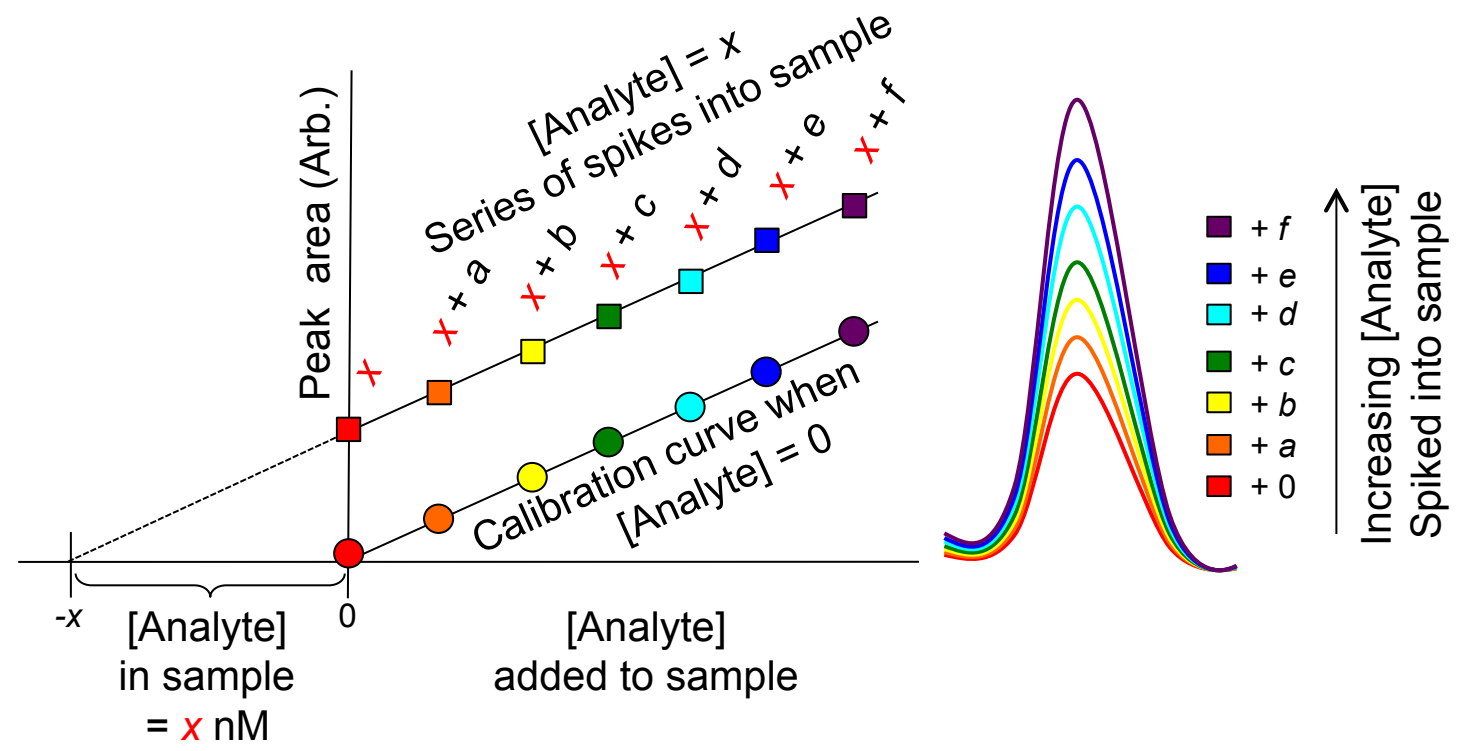

Figure 3 The standard addition method (SAM) for absolute quantification. 